\title{
Aplicação de Programação Inteira na Distribuição de Encargos Didáticos em Instituições de Ensino ${ }^{1}$
}

P.S. FERREIRA ${ }^{2}$, E.W. KARAS ${ }^{3}$, F.L. PALUCOSKI, A.A. RIBEIRO ${ }^{5}$, Departamento de Matemática, UFPR - Universidade Federal do Paraná, Cx.P. 19081, 81531-980, Curitiba, PR, Brasil.

A.L. SILVA, Departamento de Engenharia de Produção, UFPR - Universidade Federal do Paraná, 81531-980 Curitiba, PR, Brasil.

Resumo. Este trabalho propõe uma formulação matemática de programação linear binária para distribuição dos encargos didáticos em uma instituição de ensino que procura maximizar a satisfação dos professores. A formulação, em duas variantes, é implementada na designação de professores às turmas ofertadas pelo Departamento de Matemática da Universidade Federal do Paraná - UFPR. Foi desenvolvido um sistema que permite que os professores preencham on-line um questionário com suas restrições de horários e preferências por turmas. Após a coleta das informações o sistema gera as matrizes necessárias para implementar o modelo matemático. Para analisar os resultados, define-se um índice médio de satisfação dos professores.

Palavras-chave. Modelagem matemática, programação linear inteira, distribuição de encargos didáticos.

\section{Introdução}

As instituições de ensino precisam a cada período letivo estabelecer os horários das aulas e designar um professor para cada turma, procurando satisfazer as necessidades pedagógicas e as preferências individuais dos professores. Esta árdua tarefa, realizada, na maioria das instituições, manualmente, insere-se no contexto de problemas de designação [11]. O problema não é novo, mas devido às particularidades de cada instituição, assume nuances diferentes. Em [18], os autores apresentam os principais tipos de problemas de programação de horários em instituições de ensino. Em escolas de ensino fundamental e médio, normalmente, é necessário elaborar a

\footnotetext{
${ }^{1}$ Edital Universal CNPq 476043/2009-3

${ }^{2}$ pri.savulski@hotmail.com. Bolsista CNPq pelo Programa de Pós-Graduação em Matemática Aplicada

${ }^{3}$ ewkaras@ufpr.br. Bolsista Produtividade CNPq

${ }^{4}$ felipe.palucoski@gmail.com. Especialização para Professores de Matemática da UFPR

5 ademir.ribeiro@ufpr.br

${ }^{6}$ arinei@ufpr.br
} 
grade horária de disciplinas de cada turma levando em consideração a disponibilidade de horário dos professores. Neste contexto, [5, 10, 20, 21] sugerem uma formulação matemática com variáveis binárias que prioriza a satisfação dos professores. As principais dificuldades estão relacionadas com o tratamento das informações a serem consideradas na formulação e com o tempo computacional, o que motiva o uso de heurísticas. Em [3], os autores comparam soluções fornecidas através de métodos heurísticos: Simulated Annealing, Busca Tabu [17, 19, 22] e Algoritmos Genéticos, enquanto em [24, 25], o problema é resolvido através do procedimento GRASP (Greedy Randomized Adaptive Search Procedures) com refinamento através de Busca Tabu. Uma heurística de busca local baseada em caminhos mínimos é desenvolvida em [23]. Método exato e heurísticos são comparados em [7] e a sinergia entre eles é explorada em [16]. Um ambiente de apoio à construção de horário escolar pela internet é apresentado em [14].

Em instituições de ensino superior há o problema de programação de horários de cursos (course timetabling) que envolve tanto a definição do horário das aulas quanto das salas em que essas aulas serão ministradas [4, 9, 15]. Fixados os horários das aulas, precisa-se, então, designar professores para cada turma atendendo restrições de caráter pedagógico e de preferência dos professores [2, 12]. Em [8], o autor aborda o problema por Algoritmo Genético e elabora uma interface para que a modelagem possa ser aplicada em outras instituições. Este trabalho [6] propõe uma formulação matemática de programação linear binária para designação de professores às turmas em instituições em que os horários das turmas são preestabelecidos, como é o caso do Departamento de Matemática da Universidade Federal do Paraná - UFPR. Cabe observar que uma mesma disciplina pode ser ofertada em diferentes horários para diferentes cursos. Cada horário semanal fixado para cada disciplina ofertada pelo Departamento caracteriza uma "turma".

É possível adaptar a formulação proposta para outros departamentos e instituições. Na Seção 2. apresenta-se a formulação básica. O sistema para coleta e gerenciamento das informações é discutido na Seção 3.. Discute-se na Seção 4. uma variante da formulação básica que resolve o problema em duas fases. Na primeira, procura-se minimizar o número de turmas atribuídas a professores que não tinham preferência por elas. Na segunda fase, fixa-se esse número e maximiza-se a satisfação geral dos professores. Para facilitar a comparação dos resultados define-se um índice médio de satisfação. A formulação, em suas duas variantes é implementada e testada. Os resultados são discutidos na Seção 5.

\section{Formulação Básica}

Considere que $T$ turmas devem ser distribuídas para $P$ professores em $H$ horários ao longo de $D$ dias semanais. A variável de decisão binária $x(p, t)$ representa a atribuição professor - turma, ou seja, para todo $p=1, \ldots, P$ e $t=1, \ldots, T$, definese:

$$
x(p, t)= \begin{cases}1, & \text { se o professor } p \text { assume a turma } t \\ 0, & \text { caso contrário. }\end{cases}
$$

A carga horária de cada professor $p$ deve estar compreendida entre um valor 
mínimo $H_{\min }(p)$ e um valor máximo $H_{\max }(p)$ definidos pela instituição. Note que se a carga horária semanal de um professor é fixa, os valores mínimo e máximo coincidem.

Os horários de cada turma são fixados e armazenados da seguinte forma:

$$
H T(t, h, d)= \begin{cases}1, & \text { se a turma } t \text { tem aula no horário } h \text { do dia } d, \\ 0, & \text { caso contrário. }\end{cases}
$$

Cada professor $p$ atribui um peso $e(p, t)$ pela preferência em assumir a turma $t$. Para turmas que o professor não gostaria de lecionar, considera-se peso nulo. $\mathrm{O}$ objetivo do modelo é maximizar a satisfação total dos professores dada por

$$
S=\sum_{p=1}^{P} \sum_{t=1}^{T} e(p, t) x(p, t) .
$$

A formulação básica considerada é descrita abaixo:

$$
\begin{aligned}
& \text { maximizar } \quad \sum_{p=1}^{P} \sum_{t=1}^{T} e(p, t) x(p, t) \\
& \text { sujeito a } \quad \sum_{t=1}^{T} H T(t, h, d) x(p, t) \leq 1, \quad \text { para todo } p, d, h \\
& \sum_{p=1}^{P} x(p, t)=1, \quad \text { para todo } t, \\
& \sum_{t=1}^{T} \sum_{h=1}^{H} \sum_{d=1}^{D} H T(t, h, d) x(p, t) \geq H_{\min }(p), \quad \text { para todo } p, \\
& \sum_{t=1}^{T} \sum_{h=1}^{H} \sum_{d=1}^{D} H T(t, h, d) x(p, t) \leq H_{\max }(p), \quad \text { para todo } p, \\
& x(p, t)=\{0,1\}, \quad \text { para todo } p, t .
\end{aligned}
$$

As restrições (2.3) exigem que cada professor não pode assumir mais do que uma turma num mesmo horário de um determinado dia. As restrições (2.4) garantem que nenhuma turma ficará sem professor. O conjunto de restrições (2.5) e (2.6) impõem, como já dito anteriormente, que a carga horária semanal de cada professor deve estar compreendida entre valores mínimos e máximos. Finalmente, (2.7) definem as variáveis como binárias.

A formulação matemática proposta de programação linear inteira binária $[1,11]$ pode ser resolvida com o auxílio do software LINGO.

\section{Coleta dos Dados}

O armazenamento das informações para alimentar o modelo foi feito de forma com-

putacional. As informações dos horários das turmas vêm do próprio sistema de 
abertura de turmas pela instituição. Foi desenvolvido um sistema [13] que disponibiliza os horários das turmas e possibilita aos professores preencher um formulário on line com suas preferências. Tal sistema foi implementado em PHP - Hipertext Preprocessor que é uma linguagem de programação para criação e gerenciamento de páginas dinâmicas na $W e b$.

Inicialmente, o professor indica os horários semanais em que não pode assumir aulas. Assim, automaticamente o sistema zera as variáveis de decisão $x(p, t)$ para toda turma $t$ em horário incompatível com o horário do professor. Com isto, a quantidade de variáveis do problema reduz significativamente e não há risco de atribuir ao professor uma turma que ele não possa assumir.

Em seguida, o sistema apresenta ao professor $p$ apenas as turmas compatíveis com sua disponibilidade de horário para este marcar suas turmas preferidas. O professor deve, então, atribuir um peso a cada turma, de forma a refletir sua preferência em ministrar aula para aquela turma. O peso $e(p, t)$ varia do valor 0 , situação em que o professor $p$ não tem interesse algum em ministrar aula para a turma $t$, até o valor 5 , caso que indica a maior preferência. Destaca-se que um professor pode atribuir uma mesma preferência para várias turmas. O fato de o professor não ter interesse em ministrar uma turma não indica que ele não pode assumí-la.

Após o preenchimento dos formulários, o sistema gera automaticamente as matrizes para alimentar o modelo. Ou seja, o sistema fixa em zero a variável $x(p, t)$ para toda turma $t$ em horário que o professor $p$, por razões particulares, não pode assumir aula e gera as seguintes matrizes:

- $H T$ de dimensão $T \times H \times D$, cujo elemento $H T(t, h, d)$ é 1 se a turma $t$ tem aula no horário $h$ do dia $d$; caso contrário, $H T(t, h, d)=0$.

- $H_{\min }$ e $H_{\max }$ de dimensões $P \times 1$, cujos elementos $H_{\min }(p)$ e $H_{\max }(p)$ representam a carga horária semanal mínima e máxima, respectivamente, do professor $p$.

- $e$ de dimensão $P \times T$, cujo elemento $e(p, t) \in\{5,4,3,2,1,0\}$ representa o peso de preferência que o professor $p$ tem pela turma $t$.

\section{Discussão sobre a Formulação}

Discute-se nesta seção algumas particularidades na resolução da formulação básica e uma forma de analisar o resultado obtido.

\subsection{Formulação em duas fases}

Algumas turmas podem não ter sido escolhidas por nenhum professor, ou seja, pode existir alguma turma $t$ para a qual $e(p, t)$ é nula para todo professor $p$. Embora ninguém tenha interesse em assumí-la, ela precisa ser ofertada e consequentemente, atribuída a algum professor. Mesmo que não haja turmas nesta situação, ao procurar atender as restrições do problema, algumas turmas deverão ser designadas a professores que não têm qualquer preferência por elas. Além disso, maximizar a satisfação total pode fazer com que, em favor da coletividade, o número de turmas 
nesta situação aumente. A fim de minimizar este número, foram consideradas duas fases na resolução do problema.

Na primeira, considera-se as restrições da formulação básica com o objetivo de minimizar o número de turmas atribuídas com satisfação nula, o que resulta em um certo número $N$. Na segunda fase, resolve-se o modelo básico com a restrição adicional de que o total de turmas atribuídas a professores com preferência nula seja $N$.

Com essa estratégia consegue-se maximizar a satisfação global dos professores para todos os possíveis conjuntos de $N$ turmas advindas da primeira fase.

Primeira fase. O objetivo da primeira fase é minimizar a quantidade de turmas designadas a professores sem que eles as tenham escolhido. Para tanto consideramse variáveis auxiliares $r(p, t)$ definidas por

$$
r(p, t)=x(p, t)(1-\min \{1, e(p, t)\}) .
$$

Note que $r(p, t)=1$ quando a turma $t$ é atribuída a um professor $p$ que não tem interesse em assumí-la e vale zero nos demais casos. O ideal é que $r(p, t)$ seja sempre nula, o que nem sempre é possível. A soma destas variáveis quantifica o número de turmas que são atribuídas a um professor que não a tinha escolhido. É esta soma que deseja-se minimizar. Assim, na primeira fase resolvemos o problema de minimizar

$$
\sum_{p=1}^{P} \sum_{t=1}^{T} r(p, t)
$$

com as restrições da formulação básica discutida na Seção 2.. A resolução deste problema fornece um número mínimo $N$ de turmas que terão que ser atribuídas a professores que não tem qualquer preferência em assumí-las.

Segunda fase. Na segunda fase, procura-se maximizar a satisfação dos professores tendo em vista que $N$ turmas terão que ser assumidas por professores que não as solicitaram. Resolve-se então o problema de maximizar a satisfação dos professores (2.1) com as restrições da formulação básica e a restrição adicional de que a quantidade de turmas atribuídas a professores que não tem qualquer preferência em assumí-las seja $N$, ou seja,

$$
\sum_{p=1}^{P} \sum_{t=1}^{T} r(p, t)=N
$$

Resolvendo-se o problema nestas duas fases, maximiza-se a satisfação global dos professores tomando-se o cuidado de minimizar o número de professores insatisfeitos por assumir turmas que não haviam solicitado.

\subsection{Disciplinas específicas}

Em cada instituição existem, normalmente, disciplinas do ciclo básico que podem ser ministradas por qualquer professor e disciplinas do ciclo específico que devem ser ministradas por especialistas da área. Turmas correspondentes a disciplinas do 
ciclo específico devem ser atribuídas a professores que se sintam confortáveis em ministrá-las e portanto as tenham colocado entre suas preferências. Assim, para toda turma $t$ que a critério da instituição seja considerada do ciclo específico e que tenha sido escolhida por algum professor, ou seja, tenha $e(p, t) \neq 0$ para algum $p$, acrescentamos à formulação básica a restrição

$$
\sum_{\substack{p=1 \\ e(p, t) \neq 0}}^{P} r(p, t)=0, \quad \text { para toda turma } t \text { tal que } \operatorname{esp}(t)=1
$$

onde $r(p, t)$ é definido em (4.1) e

$$
\operatorname{esp}(t)= \begin{cases}1, & \text { se a turma } t \text { é do ciclo específico, } \\ 0, & \text { caso contrário. }\end{cases}
$$

Estas restrições impõem que toda turma $t$ do ciclo específico deve ser atribuída a alguém que tenha interesse em ministrá-la.

As restrições de disciplinas específicas podem ser acrescentadas na formulação básica independentemente se o problema é resolvido em uma ou duas fases.

\section{3. Índice de satisfação}

Para facilitar a análise dos resultados obtidos, considera-se um índice de satisfação médio dos professores. Suponha que ao professor $p$ foram atribuídas $q(p)$ turmas não necessariamente as de sua máxima preferência. Por outro lado, considere que as $q(p)$ turmas de sua máxima preferência sejam $t_{1}, t_{2}, \ldots, t_{q(p)}$. O índice individual de satisfação do professor $p$ é dado por:

$$
I(p)=\frac{\sum_{t=1}^{T} e(p, t) x(p, t)}{\sum_{j=1}^{q(p)} e\left(p, t_{j}\right)} .
$$

Note que $I(p)$ é a razão entre a soma dos pesos das $q(p)$ turmas designadas ao professor $p$, e o peso máximo se as $q(p)$ turmas designadas a ele fossem as de sua maior preferência. Este índice está entre 0 e 1 . Índice unitário significa que as turmas atribuídas ao professor são as de sua preferência. O índice é nulo no caso extremo em que são atribuídas ao professor apenas aulas que ele não havia escolhido, mas que se encaixam no seu horário. A média dos índices $I(p)$ fornece o índice de satisfação médio dos professores, ou seja,

$$
I=\frac{1}{P} \sum_{p=1}^{P} I(p)
$$




\section{Aplicação}

No Departamento de Matemática da UFPR, a cada semestre são distribuídas a cerca de 50 professores em torno de 100 turmas, com carga horária semanal de 3, 4 ou 6 horas. O árduo trabalho de distribuição das turmas era feito, até então, de forma manual por uma comissão que analisava a preferência e disponibilidade de horário de cada professor.

A formulação sugerida neste trabalho foi aplicada na distribuição dos encargos didáticos do primeiro e do segundo semestre de 2010 do Departamento de Matemática da UFPR, onde foram distribuídas $T=103$ turmas de graduação no primeiro semestre e $T=94$ no segundo semestre para $P=44$ professores em $H=15$ horários em cada um dos $D=5$ dias semanais. Os problemas foram resolvidos em um computador Core 2 duo 8500, 2.27 GHz com 4Gb de memória RAM e Windows $\mathrm{XP}$, com o auxílio do software LINGO 9.

Análise dos resultados. Os problemas foram resolvidos de duas formas. Primeiro, através da formulação básica descrita na Seção 2.. Em seguida, pela decomposição em duas fases descrita na Seção 4.. A tabela a seguir sintetiza os resultados obtidos. A coluna $S$ fornece a satisfação global dos professores dada por (2.1), enquanto a coluna seguinte apresenta o índice de satisfação médio dado por (4.3). Por outro lado, a penúltima coluna fornece o número $N$ de turmas designadas a professores que não as solicitaram. Finalmente, a última coluna dá o tempo computacional, em segundos, gasto para resolver cada um dos problemas.

Tabela 1: Resultados

\begin{tabular}{|l|l|c|c|c|c|}
\hline Semestre & Formulação & $S$ & $I$ & $N$ & CPU \\
\hline \hline Primeiro & Básica & 394 & 0,8252 & 13 & 8 \\
\hline Primeiro & Duas fases & 363 & 0,7477 & 7 & 16 \\
\hline \hline Segundo & Básica & 373 & 0,8155 & 12 & 8 \\
\hline Segundo & Duas fases & 368 & 0,8064 & 8 & 22 \\
\hline
\end{tabular}

A satisfação global e o índice de satisfação médio são maiores quando o problema é resolvido através da formulação básica em uma única fase. Por outro lado, em favor da coletividade, mais turmas são atribuídas a professores que não as solicitaram. Note que, embora os problemas sejam resolvidos por método exato, o tempo computacional é baixo.

Foi feita também uma interface gráfica com o auxílio do software Visual Basic, que transforma a resposta fornecida das turmas atribuídas a cada professor em planilhas e tabelas que podem ser facilmente analisadas pela instituição.

Após a análise dos resultados, adotou-se a solução fornecida pela resolução em duas fases que se preocupa em reduzir o número de professores insatisfeitos.

Uma crítica ao resultado é de que alguns professores ficaram com turmas espalhadas pela semana com grandes janelas durante o dia. Deve-se, num próximo trabalho, melhorar a formulação de modo a reduzir tais situações, procurando con- 
centrar os horários de aulas de cada professor. Além disso, pretende-se equilibrar os índices de satisfação individual.

\begin{abstract}
We propose a binary linear programming model to distribute the classes to the instructors maximizing their satisfaction. The model was implemented for distributing the classes to the professors of Department of Mathematics at Federal University of Paraná. We also developed an on-line system where the professors inform their unavailable work time and their preferred classes. After having all information the system generates the matrices to implement the model. In order to analyze the results we define a mean satisfaction index.
\end{abstract}

\title{
Referências
}

[1] D. Bertsimas, J.N. Tsitsiklis, "Introduction to Linear Optimization", Athena Scientific, USA, 1997.

[2] E.K. Burke, S. Petrovic, Recent research directions in automated timetabling, European Journal of Operational Research - EJOR, 140, No. 2 (2002), 266-280.

[3] A. Colorni, M. Dorigo, V. Maniezzo, Metaheuristics for high school timetabling, Computational Optimization and Applications, 9 (1998), 275-298.

[4] A.A. Constantino, W. Marcondes Filho, D. Landa-Silva, Iterated heuristic algorithms for the classroom assignment problem, In "Proceedings of the 8th International Conference on the Practice and Theory of Automated Timetabling - PATAT", p. 152-166, 2010.

[5] D.M.B. Costa, "Distribuição das Cargas Horárias de Professores em uma Instituição de Ensino", Monografia do Curso de Especialização em Matemática Aplicada, UFPR, 1994.

[6] P.S. Ferreira, E.W. Karas, F.L. Palucoski, A.A. Ribeiro, A.L. Silva, Aplicação de programação inteira na distribuição de encargos didáticos em instituições de ensino, Anais do XXXIII CNMAC - Congresso Nacional de Matemática Aplicada e Computacional, Águas de Lindóia, SP, Setembro 2010.

[7] A.R.T. Góes, "Otimização na Distribuição da Carga Horária de Professores: método exato, método heurístico, método misto e interface", Dissertação de Mestrado, UFPR, 2005.

[8] O.O. Braz Jr, "Otimização de Horários em Instituições de Ensino Superior através de Algoritmos Genéticos", Dissertação de Mestrado, UFSC, 2000.

[9] P. Kostuch, The university course timetabling problem with a 3-phase approach, In "Proceedings of the 5th International Conference on the Practice and Theory of Automated Timetabling - PATAT", p. 251-266, 2004.

[10] E.G.S. Kotsko, "Otimização na Construção da Grade Horária Escolar: uma aplicação", Dissertação de Mestrado, UFPR, 2003. 
[11] L.L. Lapin, "Quantitative Methods for Business Decisions", Wadsworth Publishing Company, USA, sixth edition, 1996.

[12] B. McCollum, A perspective on bridging the gap between theory and practice in university timetabling, In "Proceedings of the 6th International Conference on the Practice and Theory of Automated Timetabling - PATAT", vol. 3867, p. 3-23. Springer-Verlag Lecture Notes in Computer Science, 2006.

[13] F.L. Palucoski, "Um Sistema para Coleta e Processamento de Dados para a Distribuição de Encargos Didáticos", Monografia do curso de Especialização para Professores de Matemática, UFPR, 2009.

[14] P.R. Pinheiro, J.A. Oliveira, Um ambiente de apoio a construção de horário escolar na WEB: modelagem, implementação e aplicação nas escolas de ensino médio, In "XXXIII Simpósio Brasileiro de Pesquisa Operacional", Campos do Jordão, SP, 2001.

[15] H. Rudová, T. Müller, K. Murray, Complex university course timetabling, Journal of Scheduling, 2010. DOI: 10.1007/s10951-010-0171-3.

[16] H.G. Santos, "Formulações e Algoritmos para o Problema de Programação de Horários em Escolas", Tese de Doutorado, UFF, 2007.

[17] H.G. Santos, L.S. Ochi, M.J.F. Souza, A Tabu Search heuristic with efficient diversification strategies for the class/teacher timetabling problem, $A C M$ Journal of Experimental Algorithmics - JEA, 10, No. 2 (2006).

[18] H.G. Santos, M.J.F. Souza, Programação de horários em instituições educacionais: formulações e algoritmos, In "XXXIX SBPO - Simpósio Brasileiro de Pesquisa Operacional", No. 1, p. 2827-2882, Fortaleza, 2007.

[19] H.G. Santos, M.J.F. Souza, L.S. Ochi, An efficient Tabu Search heuristic for the school timetabling problem, Lecture Notes on Computer Science - LNCS, 3059 (2004), 468-482.

[20] H.G. Santos, E. Uchoa, L.S. Ochi, N. Maculan, Strong bounds with cuts and column generation for class-teacher timetabling, In "Proceedings of the 7th International Conference on the Practice and Theory of Automated Timetabling - PATAT", 2008. DOI 10.1007/s10479-010-0709-y.

[21] H.G. Santos, E. Uchoa, L.S. Ochi, N. Maculan, Strong bounds with cut and column generation for class-teacher timetabling, Annals of Operations Research, to appear.

[22] V.N. Sousa, A.C. Moretti, V.A. Podestá, Programação da grade de horário em escolas de ensino fundamental e médio, Pesquisa Operacional, 28, No. 3, (2008), 399-421.

[23] M.J.F. Souza, "Programação de Horário em Escolas: uma aproximação por metaheurísticas", Tese de Doutorado, UFRJ, 2000. 
[24] M.J.F. Souza, N. Maculan, L.S. Ochi, Uma heurística para a programação de horários em escolas, TEMA - Tend. Mat. Apl. Comput., 2, No. 1 (2001), $213-222$.

[25] M.J.F. Souza, N. Maculan, L.S. Ochi, A GRASP - TABU SEARCH algorithm for solving school timetabling problems, volume 15, chapter 31, pages 659-672. Combinatorial Optimization Book Series, Metaheuristics: Computer Decision - Making, 2003. 